\title{
Interfacial Bonding Mechanism and Mechanical Performance of Ti/Steel Bimetallic Clad Sheet Produced by Explosive Welding and Annealing
}

\author{
Zu Guoyin, Sun Xi, Zhang Jinghua
}

Northeastern University, Shenyang 110189, China

\begin{abstract}
The microstructural characterization and interfacial shear tests of Ti/Steel bimetallic clad sheets were taken to study the interfacial bonding mechanism during the explosive welding process. The results show that the wavy interface with several vortexes forms between the dissimilar metal matrixes due to the interface deformation. A significant metal flow of steel matrix caused by the severe plastic deformation induces the continuous vortexes in the interface. The TEM and XRD analyses clarify that the nanoscale interfacial interlayer contains solid solutions and a small amount of intermetallic compounds. The shear strength of the interface along the explosive welding direction is enhanced by the wavy interface. The fracture of the bonding interface presents the ductile features on the vortex while brittle features on the smooth interface.
\end{abstract}

Key words: clad sheet; explosive welding; interface; TEM; intermetallic compounds

The clad sheets composed of dissimilar metals have been attracting much attention by combining the outstanding properties of dissimilar metals ${ }^{[1,2]}$. Particularly, the explosive welding technique is popular in the cladding field for its good capability of large deformation and high strain energy ${ }^{[3]}$. The study on the bond strength of explosive welded specimens reveals that, during explosive welding process, the interface is molten and has a remarkable atomic diffusion under the significant explosive impacting and deformation energy ${ }^{[4]}$.

A good metallic bonding can be formed in the interface based on the control of explosive parameters ${ }^{[5]}$. After that, thermal annealing is necessary to remove the explosive residual work hardening ${ }^{[6]}$. A series of clad sheets can be produced by the explosive welding technique through designing the component material and thickness ratio ${ }^{[7]}$. In particular, Ti/Steel clad sheets were manufactured by Kundu, which can be applied in the heat-exchange facility based on the excellent heat conduction, mechanical strength, and corrosion resistance as well as low cost ${ }^{[8]}$.
Nowadays, the microstructural characterization of the bonding interface has been wildly studied in the metallographic approach. Gulenc investigated the interfacial properties and weldability of aluminum and copper plates by the explosive welding method ${ }^{[9]}$. Kacar and Acarer reported the relationship between microstructure and mechanical properties of explosive welded duplex stainless steel-steel ${ }^{[10]}$. Kahraman systematically investigated the interfacial bonding quality of clad sheets using the tensile shear, bending and hardness tests ${ }^{[11]}$. The effect of explosive parameters on the cladding product has been summarized to guide the production in the future. The numerical simulation of explosive welding process has been reported to clearly understand the welding mechanism ${ }^{[12]}$. In addition, Acarer and Demir have studied the fundamental welding process and thermal kinetics in the explosive welded aluminum-dual phase steel ${ }^{[13]}$. However, the interfacial microstructure and fracture have not been deeply studied through the electron microscopy. The bonding mechanism of the welded interface in the explosive process

Received date: April 25, 2016

Foundation item: National Natural Science Foundation of China (50971038)

Corresponding author: Zu Guoyin, Ph. D., Professor, School of Materials Science and Engineering, Northeastern University, Shenyang 110819, P. R. China, Tel: 0086-24-83686462, E-mail: zugy@smm.neu.edu.cn 
is also lack of clear interpretation.

In the present paper, the interfacial microstructure and chemical phase were characterized through the optical and electron microscopy, and the X-ray diffraction analysis. The interfacial interlayer in high magnification was observed by the transmission electron microscope (TEM). The interface effect on the shear performance of explosive welded Ti/Steel clad sheets were studied according to the shear tests on the interface along the explosive direction.

\section{Experiment}

The raw materials were steel sheet SA516Gr.70 with thickness of $72 \mathrm{~mm}$ and titanium sheet SB265Gr.2 with thickness of $12 \mathrm{~mm}$. They were fully annealed and obtained the initial shear strength of 265 and $200 \mathrm{MPa}$, respectively. They were assembled with a parallel distance of $12 \mathrm{~mm}$. The powdery emulsion explosives with density of 0.84 $\mathrm{g} \cdot \mathrm{cm}^{-3}$ and thickness of $41 \mathrm{~mm}$ exploded from the center of clad layer at the velocity of $2460 \mathrm{~m} \cdot \mathrm{s}^{-1}$. Then the clad sheets were heat treated at $540{ }^{\circ} \mathrm{C}$ for $3 \mathrm{~h}$. The metallographic preparation was taken on the longitudinal section along the explosive direction following the conventional methods.

The overall interfacial microstructures of the explosive welded clad sheet were observed by optical microscope (OM) and scanning electron microscope (SEM) SSX-550 equipped with energy dispersive $\mathrm{X}$-ray detector (EDX). The phase constitution of the interfacial zone was measured through XRD analysis on the X'Pert Pro instrument with $\mathrm{Cu}$ target. Particularly, the interfacial characteristic was verified by the G2 TECAN TEM with a $200 \mathrm{kV}$ accelerating voltage on the cross-sectional film which was thinned by precision ion milling.

The compression shear tests were carried out on the SANSCMT 5000 materials testing system at room temperature with the loading stress rate of $4 \mathrm{MPa} \cdot \mathrm{s}^{-1}$. The load was taken along the interface. The samples with shear area of $5 \mathrm{~mm} \times 25 \mathrm{~mm}$ were prepared from the center of clad sheet along the explosive direction according to ASTM 264. The compression shear schematic diagram is given in Fig.1. After that, the fracture observation was observed by SEM to analyze the interfacial failure.

\section{Results and Discussion}

\subsection{Interfacial microstructure of $\mathrm{Ti} / \mathrm{steel}$ clad sheet}

The Ti/Steel clad sheet, obtaining the significant workhardening during the explosive welding process, must be annealed to get the optimal mechanical properties. In our previous investigations, applicable annealing parameters had been gotten and used in this experiment. Thus, only the annealed clad sheets are studied in the following sections. The interfacial zone of explosive welded Ti/Steel clad sheet after annealing is illustrated in Fig.2. It is revealed that some distinct wavy vortexes of steel exist on the interface

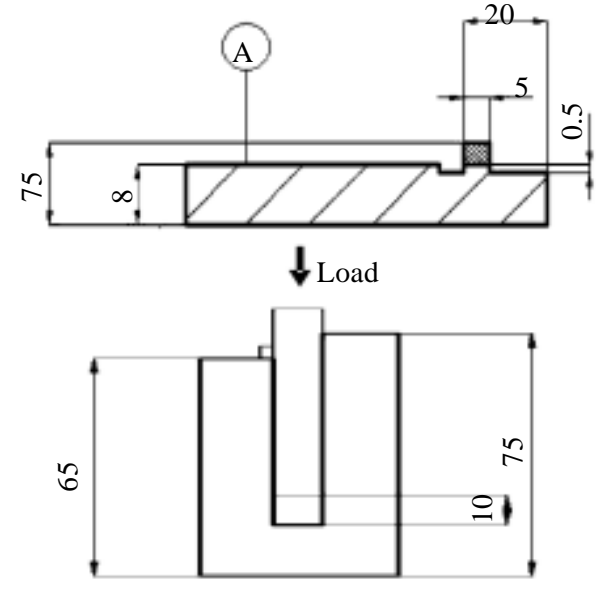

Fig. 1 Schematic diagram of the compression shear test for clad sheet

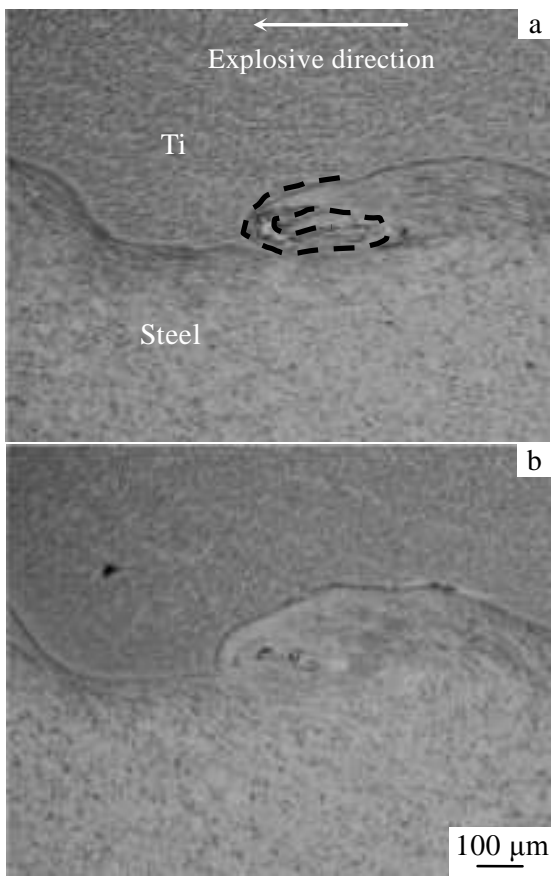

Fig.2 Interfacial macrostructure of Ti/Steel clad sheet: (a) clear path is illustrated by the dotted line in the interface and (b) a few of black molten objects are wrapped into the interfacial vortex

with the elongated grains along the explosive direction.

As illustrated by the dotted line track in Fig.2, the whole deformation in the interface is clear according to the distribution of vortex. During the explosive welding process, the substrate steel plate gets a significant explosive impacting, which causes a severe deformation on the metal surface. Due to the lower melting temperature of steel than that of titanium, the deformation heat can soften steel, and then the surface layer of steel gets a prominent metal flow 
along the explosive direction. Also along with the severe plastic deformation, the grains on the bonding interface are refined as shown in Fig.2. It is indicated in Fig.2b that a few of black molten objects are wrapped into the interfacial vortex. It can be ascribed to the molten contaminants on the metal surface, which are drawn in the interfacial vortex by the explosive deformation.

The magnification of interface is shown in Fig.3, with the track of metal flow along the dotted line in Fig.3a. It is seen that the stream line is discontinuous in the vortex, illustrated as the label of 1 . Generally, the surface layer of steel is elongated along the explosive direction, shown by the flow line in Fig.3b. Under the impacting pressure, the surface layer of steel substrate sputters forwardly. Under the limit of $\mathrm{Ti}$ sheet, the steel sputtering is hindered and wrapped into its substrate. As well, a few of surface inclusions are mixed into the vortex. During the sputtering process, the metal flow may be discontinuous because of the small sputtering.

The chemical composition is simply revealed according to the SEM and EDX. Fig.4 reveals the SEM microstructure of interfacial zone of clad sheet. It is seen that the grain size of interfacial steel is about $3 \sim 5 \mu \mathrm{m}$ for the severe deformation during explosive welding. The stream line of steel and some inclusions of titanium or other contaminations in the interfacial vortex are also observed. The interfacial wave clarifies the explosive process in terms of impulse wave. The composition distribution is roughly revealed by the backscattered electron mode SEM (BSEM) in Fig.4c. Some interfacial phases exist on the bonding interface. It is seen that the distribution of interfacial phase is inhomogeneous along the interface for the complicated deformation condition in the bonding interface. In order to clarify the interfacial phase, TEM observation is carried out on the interfacial zone.

According to the SEM microstructure in Fig.4c, the interfacial composition needs to be measured to clarify the chemical phase transformation during explosive welding. XRD analysis shows (in Fig.5) the existence of some intermetallic compounds of FeTi. During the explosive welding process, a great deal of deformation energies accumulates on the interface. After the thermal annealing, the interfacial phase could be formed based on the thermal diffusion and phase transformation. According to the Ti-Fe binary alloy phase diagram, it is possible for the formation of several intermetallic compounds, such as $\mathrm{FeTi}$ and $\mathrm{Fe}_{2} \mathrm{Ti}$, when the annealing temperature is above $500{ }^{\circ} \mathrm{C}$. In present work the $540{ }^{\circ} \mathrm{C}$ annealing makes the limited distance of atomic diffusion in the bonding interface between $\mathrm{Ti}$ and Steel matrix. There is only FeTi phase formed in the chemical composition range of near atomic percent $\mathrm{Cu}_{50} \mathrm{Ti}_{50}$ according to the phase diagram ${ }^{[14]}$. Therefore, the thermodynamic condition could meet the formation of intermetallic compounds FeTi.

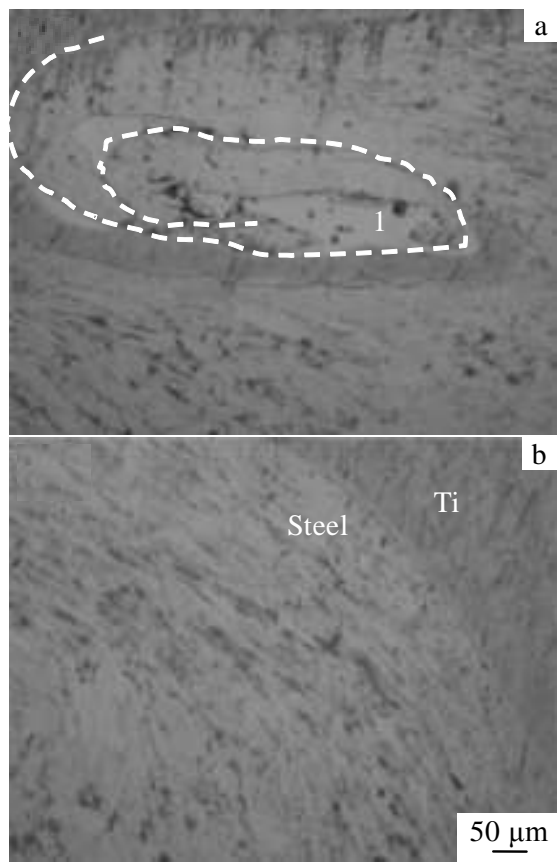

Fig. 3 High magnification of the interfacial vortex (a) and the metal flow line (b) of Ti/Steel clad sheet
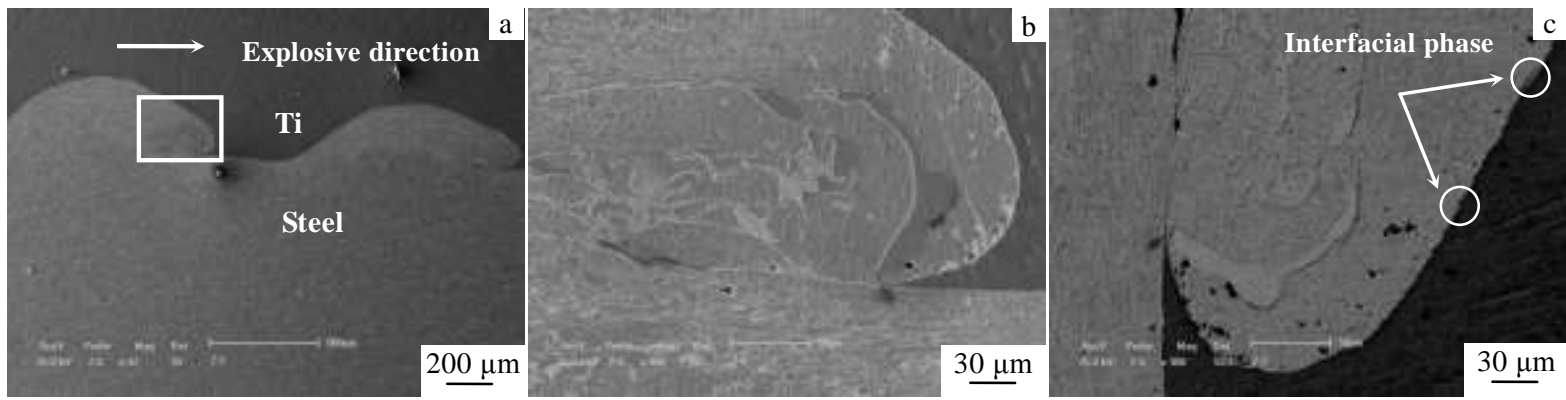

Fig. 4 SEM microstructures of the interfacial zone of Ti/Steel clad sheet: (a, b) secondary electron mode; (c) backscattered electron mode 


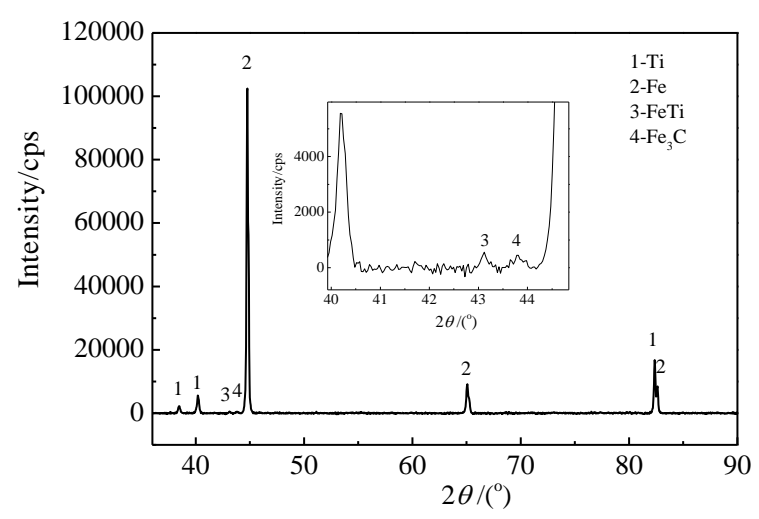

Fig. 5 XRD pattern of clad sheet after annealing (the inset is pattern corresponding to $2 \theta=40^{\circ} \sim 45^{\circ}$ )

\subsection{TEM microstructure and chemical phase of bonding interface}

The high resolution microstructure of the smooth interface far away from the vortex observed by TEM is shown in Fig.6. A clear interfacial interlayer indicated by the dotted line in Fig.6a forms between the component metal layers. The grain beside interlayer is in accordance with the SEM results in Fig.4. Some grains in submicron scale form next to the interlayer in Fig.6b for the severe deformation in the explosive process. The thickness of interfacial interlayer is $0.2 \mu \mathrm{m}$. The high magnification TEM micrograph indicates that a nanoscale sublayer shown by the arrow in Fig.6c is formed adjacent to the submicron grain of titanium sheet, which may be the amorphous structure. Yang has verified the amorphous interface of explosive welded clad sheet by the HRTEM ${ }^{[15]}$. Owing to this thin interfacial layer, a good metallurgical bonding between Ti and steel sheet is obtained.

The chemical composition of interlayer is presented by the EDX equipped on the transmission electron microscope. It is Fe-rich constitution and Ti-rich constitution for the point 1 and 2 in Fig.6b, respectively. The results give a clear evidential existence of the interfacial interlayer between dissimilar metal matrixes. The selected area electron diffraction (SAED) pattern of interfacial interlayer is indicated (in Fig.6f) the bcc structure of $\alpha$-Ti. The near-ring diffraction spots are found in the SAED, which can be ascribed to the nanoscale Ti grains in the interface after explosive welding. This result is different from the XRD analysis, revealing a few FeTi in the interfacial layer. It may be attributed to the selective deviation caused by the much smaller interlayer than the selected area aperture during the diffraction operation.

\subsection{Mechanical performance and fracture analysis}

The compression shear tests were conducted to evaluate the interfacial bonding strength. It is measured as $334 \mathrm{MPa}$

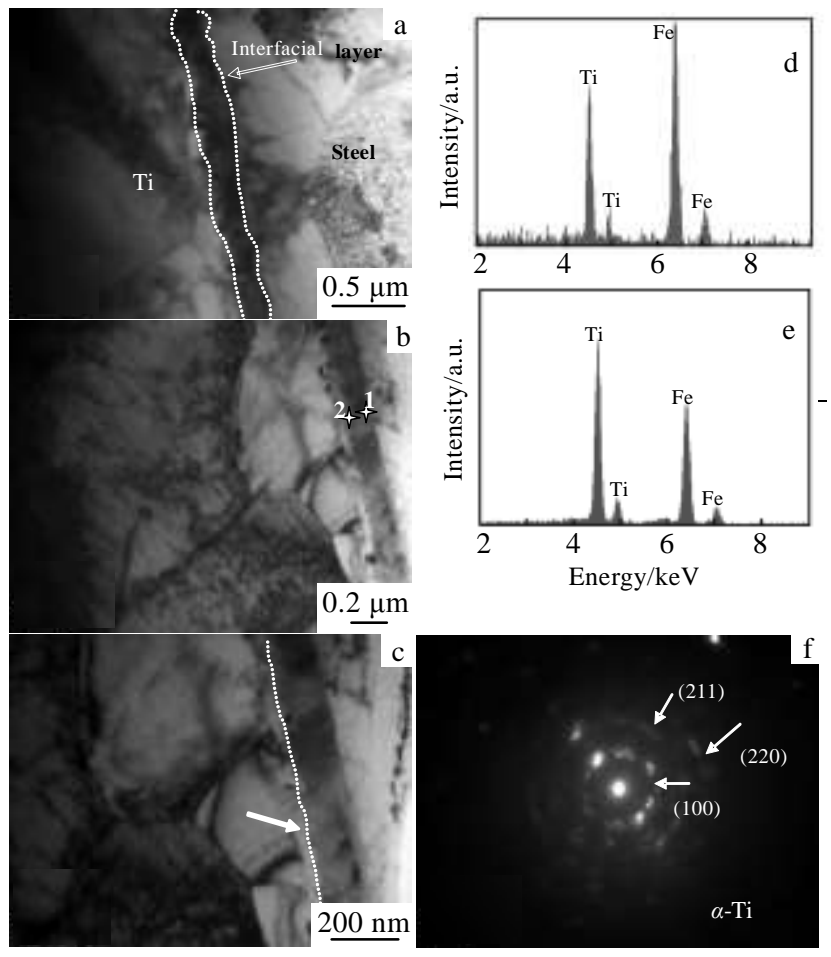

Fig. 6 TEM images (a, b) of the interfacial zone of Ti/Steel clad sheet, (c) is the magnification of the interface in Fig.6b; (d) and (e) corresponding to point 1 and 2 in Fig.6b; (f) is the selected-area diffraction pattern of the interfacial layer

for the interfacial shear strength between $\mathrm{Ti}$ and steel sheet. The fracture occurs along the bonding interface. It is found that the interfacial shear strength is drastically increased compared with the component metal layer. Based on the interfacial microstructure, the reason may be that the wavy interface presents a complicated structure and restrains the stress during the shear test. Due to the wavy interface remarkably restraining the shear stress, the interfacial strength is higher than that of the component metal. The interfacial structure is proved to be crucial to the shear strength of clad sheet, whereas the smooth interface represents the weak shear strength ${ }^{[16]}$.

In Fig.7, the shear fracture was observed by SEM to analyze the interfacial failure of clad sheet. It is seen from Fig.7a that some peaks and troughs are alternately distributed on the Ti matrix, which is good agreement with the interfacial microstructure got from the metallographic observation. A couple of peak and trough is magnified in Fig.7b, indicative of a clear difference between the microstructure. It reveals a lot of dimples and shear bands on the trough, while a river-like brittle fracture on the peak. 



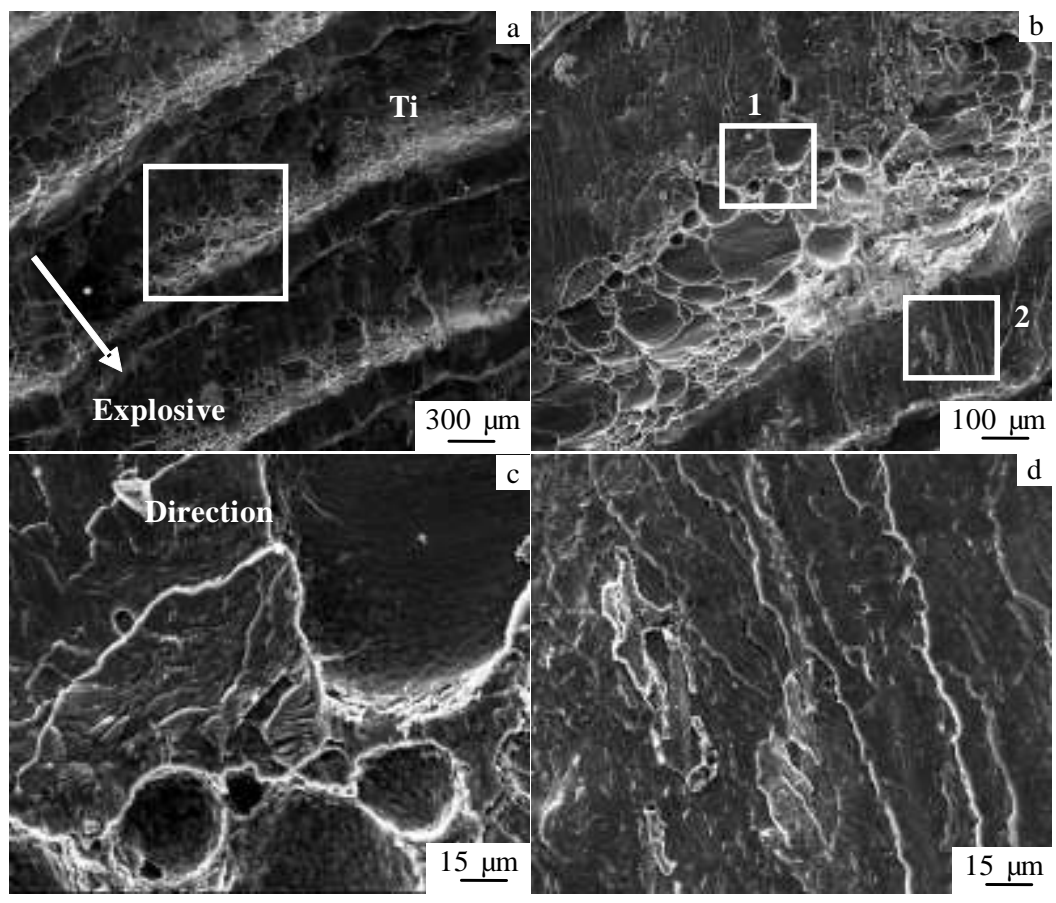

Fig. 7 Fracture morphologies of Ti matrix after the shear test of Ti/Steel clad sheet (a, b); (c) and (d) the high magnification of the selected zone 1 and 2 in Fig.7b

With the comparison of the SEM microstructure between Fig.4 and Fig.7, it can be found that the peak on Ti matrix corresponds to the interfacial vortex of steel matrix. Owing to the complicated structure, the vortex can resist the transmission of shear stress and hinder the formation of microcracks on the interface. Thus, the vortexes caused by the significant metal flow present the ductile fracture and play a good role to the shear performance of the clad sheet. The delamination of the bonding interface with the vortex is inhibited and induces a visible ductile tearing.

Seen from the high-magnification in Fig.7c, a few voids exist around the dimples, which is very likely to the explosive inclusion. The smooth surface in Fig.7d shows a distinct feature about the weak bonding. During the explosive welding, the smooth interface locally forms due to the limited metal flow caused by the explosive impulse. The surface layer is elongated and compacted by the explosive impacting. Except that, there are no other factors such as molten diffusion and additional deformation. Thus, the smooth interface with a nanoscale interlayer is formed between the dissimilar metals after thermal annealing. It is weak to resist the shear stress and presents the smooth fracture.

\section{Conclusions}

1) $\mathrm{Ti} / \mathrm{Steel}$ clad sheet are prepared by explosive welding and annealing. A wavy interface forms between the dissimilar metals due to the existence of vortexes caused by the significant metal flow and sputtering of the steel matrix for the severe impacting during the explosive welding process.

2) The interfacial zone is obtained and provides a clear evidence of interfacial interlayer. A nanoscale amorphous layer form adjacent to the titanium matrix with some submicron grains in the interface.

3) The shear strength of clad sheet along the explosive welding direction is enhanced by the stress restrain from wavy interface. The interfacial vortex improves the interfacial bonding and hinders the interfacial delamination under shear stress.

\section{References}

1 Li Y P, Zhang G P, Wang W et al. Scripta Materialia[J], 2007, 57: 117

2 Xia H B, Wang S G, Ben H F. Materials \& Design[J], 2014, 56: 1014

3 Findik F. Materials \& Design[J], 2011, 32: 1081

4 Mousavi S A A A, Al-Hassani S T S, Atkins A G. Materials \& Design[J], 2008, 29: 1334

5 Wu J P, Yang Y L, Zhao H Z et al. Materials \& Design[J], 2015, 65: 1100

6 Honarpisheh M, Asemabadi M, Sedighi M. Materials \& Design[J], 2012, 37: 122

7 Raabe D, Choi P P, Li Y J et al. MRS Bulletin[J], 2010, 35: 
982

8 Kundu S, Ghosh M, Chatterjee S. ISIJ International[J], 2004, 44: 1882

9 Gulenc B. Materials \& Design[J], 2008, 29: 275

10 Kacar R, Acarer M. Materials Science and Engineering A[J], 2003, 363: 290

11 Kahraman N, Gulenc B, Findik F. Journal of Materials Processing Technology[J], 2005, 169: 127

12 Li X J, Mo F, Wang X H et al. Science And Technology of
Welding and Joining [J], 2012, 17: 36

13 Acarer M, Demir B. Materials Letters[J], 2008, 62: 4158

14 Yang Y, Wang B F, Xiong $\mathrm{J}$ et al. Metallurgical and Materials Transactions A[J], 2006, 37A: 3131

15 Yang Y, Wang B, Xiong J. Journal of Materials Science[J], 2006, 41: 3501

16 Wang J, Misra A. Current Opinion in Solid State \& Materials Science $[\mathrm{J}], 2011,15: 20$

\title{
钛/钢爆炸复合板界面连接机理与力学行为
}

\author{
祖国胤、孙 溪、张精华 \\ (东北大学，辽宁 沈阳 110819)
}

\begin{abstract}
摘 要: 表征了钛/钢爆炸焊接复合板的界面组织和剪切性能, 研究了爆炸复合过程的界面结合机理。结果表明, 在金属板之间形成了 波状界面组织, 剧烈塑性变形造成钢基体发生明显的塑性流动, 并在界面形成连续的漩涡特征。TEM和XRD检测证实界面存在纳米尺 度的过渡层, 并包含有固溶体组织和少量金属间化合物。波状界面组织改善了复合板沿爆炸方向的界面剪切强度, 剪切断口显示漩浴组 织发生韧性断裂特征。
\end{abstract}

关键词：复合板；爆炸焊接；界面; TEM；金属间化合物

作者简介：祖国胤，男，1977 年生，博士，教授，东北大学材料科学与工程学院，辽宁 沈阳 110819, 电话: 024-83686462, E-mail: zugy@smm.neu.edu.cn 